\title{
含源汇非定常对流扩散问题 紧致四阶差分格式
}

\author{
杨 志 峰 \\ 陈国谦 \\ (中国科学院力学研究所, 北京 100080)
}

(北京师范大学环境科学研究所水环境模拟国家重点实验室, 北京 100875)

\section{关钺词对流扩散、系致差分、综合变换、非定常、计算流体力学}

对流扩散乃流体流动与传热传质的基本过程. 作为提高其数值模拟可靠性的根本途径, 对流扩散方程的高精度数值方法愈受重视 ${ }^{[1-9]}$. 其中紧致差分 (compact difference) 以其实 用、省时和高整体精度等特性倍受青睐 ${ }^{[4-9]}$. Dennis ${ }^{[9]}$ 四阶紧致格式,一维情况可推广至非定 常问题, 但处理较繁; 更为遗憾地是其格式未能充分反应对流的“迎风”效应, 不适用于对流占 优问题 ${ }^{[0 .}$. 新近发展的摄动四阶紧致差分格式 ${ }^{[6.7]}$ 可以确保对流的迎风性, 相当程度上克服了 Dennis 格式中的上述困扰, 目前尚待推广于非定常问题. 杨志峰等 ${ }^{[8]}$ 发展了非定常对流扩散 方程四阶紧致差分格式, 时间单步、空间仅含相邻结点 (即三点式), 取得了较为满意的成果. 未失一般性, 在应用于非线性对流或含有源汇问题时,亦不能确保具原有较高精度 ${ }^{[8,10-12]}$. 杨 志峰等 ${ }^{[10-12}$ 曾以含源扩散方程和泊松方程为例, 发展了对源汇项的离散化方法, 从而保证了非 济次方程差分格式亦可具有其齐次方程差分格式的精度 ${ }^{[0-12]}$. 鉴于此, 本文进一步完成如下 工作.

\section{1 对流的综合变换}

一维含源汇非定常对流扩散问题一般数学描述为

$$
\frac{\partial \phi}{\partial t}+U(x, t) \frac{\partial \phi}{\partial x}=D \frac{\partial^{2} \phi}{\partial x^{2}}+S(x, t),
$$

其中 $\phi$ 为待求量, $U 、 S$ 分别为对流流速和源汇函数, 这里为 $x 、 t$ 的函数, $D$ 是扩散系数.

$\phi-U, D-$ 运动粘性系数 $v$ 时, 式 (1) 为非线性对流扩散 (Burgers) 方程, 通常视为流 体流动 (Navier-Stokes 方程) 的模型方程.

$\phi=$ 污染浓度, $U=$ const. 时, 式 (1) 为线性的含源对流扩散方程, 在具有污染源的大 气、地表水和地下水环境及化工流动等领域中经常涉及.

兹设

$$
\downarrow(x, t)=e(x, t) \psi(x) p(t),
$$

1992-05-20 收稿，1992-09-18 收修放稕. 
代人方程 (1) 得

$$
\begin{gathered}
\phi\left(\varphi \frac{\partial \theta}{\partial \tau}+\theta \frac{\mathrm{d} \varphi}{\mathrm{d} t}\right)+U \varphi\left(\psi \frac{\partial \theta}{\partial x}+\theta \frac{\mathrm{d} \psi}{\mathrm{d} x}\right) \\
-D_{\varphi}\left(\psi \frac{\partial^{2} \theta}{\partial x^{2}}+2 \frac{\partial \theta}{\partial x} \frac{\mathrm{d} \psi}{\mathrm{d} x}+\theta \frac{\mathrm{d}^{2} \psi}{\mathrm{d} x^{2}}\right)+S,
\end{gathered}
$$

欲消除关于 $\theta$ 的对流因子和反应项,须令

$$
\begin{aligned}
& U \psi=2 D \frac{\mathrm{d} \psi}{\mathrm{d} x}, \\
& \psi \frac{\mathrm{d} \varphi}{\mathrm{d} t}+U_{\varphi} \frac{\mathrm{d} \psi}{\mathrm{d} x}=D_{\varphi} \frac{\mathrm{d}^{2} \psi}{\mathrm{d} x^{2}},
\end{aligned}
$$

这样方程 (3) 简化为

$$
\frac{\partial \theta}{\partial t}=D \frac{\partial^{2} \theta}{\partial x^{2}}+F(x, \imath),
$$

这里

$$
F(x, t)=\phi^{-1} \varphi^{-1} S .
$$

我们称式 (6) 为对流扩散方程 (1) 的等价扩散方程, $\theta$ 即为等价扩散变量.

由式 (4) 得

$$
\phi(x)=\exp \left[\frac{1}{2} \int \frac{U}{D} \mathrm{~d} x\right]
$$

代人式(5) 有

$$
\varphi(t)=\exp \left[\frac{1}{2} \int\left(\frac{\partial U}{\partial x}-\frac{D}{2}\left(\frac{U}{D}\right)^{2}\right) \mathrm{d} t\right] .
$$

我们称 $\psi(x) \varphi(t)$ 为非定常对流扩散方程 (1) 的综合变换, 其中 $\boldsymbol{\phi}(\boldsymbol{x})$ 为对流的“迎风”函数、 $\varphi(t)$ 为对流的“历经”函数. 变换 $\phi(x) \varphi(t)$ 将对流扩散型方程 (1) 变换为等价扩散型方程 (6), 较难处理的对流项不再出现, 其作用转嫁给变换函数. 故而通过式 (6) 来间接获取对流 扩散量的高阶算式要比直接由式 (1) 建立对流扩散量的高阶算式方便和简易.

\section{2 四阶紧致善分格式}

杨志峰等 ${ }^{[10,11]}$ 发展了形如式 (6) 含源汇扩散方程四阶二层三点差分格式:

$$
\begin{aligned}
2(6 r+5) \theta_{i} & =(6 r-1)\left(\theta_{i-1}+\theta_{i+1}\right)+2(5-6 r) \theta_{i}^{0} \\
& +(6 r+1)\left(\theta_{i-1}^{0}+\theta_{i+1}^{0}\right)+C_{p} F_{i}+\delta F_{i},
\end{aligned}
$$

式中下标 $i$ 表示差分点号, 上标’表示上一时段; $r=D \tau / h^{2}$, 其中 $\tau 、 h$ 为时、空步长; $C_{F}$ 为源 项差分系数, $\delta_{F}$ 为高阶修正量 ${ }^{[0]}$,具体可描述为

$$
\begin{aligned}
& C_{F}=12 r h^{2}, \\
& \delta F_{i}=(6 r+1) \tau h^{2}\left(\frac{\partial^{2} F}{\partial x^{2}}\right)_{i}-6 \tau^{2}\left(\frac{\partial F}{\partial t}+D \frac{\partial^{2} F}{\partial x^{2}}\right)_{i} .
\end{aligned}
$$

在差分子域内, $\phi 、 \varphi$ 可定积分表示为

$$
\begin{aligned}
& \varphi(\zeta)=\exp \left[\frac{1}{2} \int_{0}^{\zeta} \frac{U}{D} \mathrm{~d} x\right], \\
& \varphi(\eta)=\exp \left[\frac{1}{4} \int_{\eta}^{0}\left(\frac{U^{2}}{D}-2 \frac{\partial U}{\partial x}\right) \mathrm{d} t\right],
\end{aligned}
$$


这里 $\zeta 、 \eta$ 为差分子域内空间和时间局部坐标, $\zeta \in[-h, h] 、 \eta \in[-\tau, 0]$.

据式 (2)、(13) 和 (14), 以 $\theta(\zeta, \eta)=\phi(\zeta, \eta) \psi^{-1}(\zeta) \varphi^{-1}(\eta)=\phi(\zeta, \eta) \phi(-\zeta) \varphi(-\eta)$ 及 式 (7) 代人式 (10)-(12), 可得对流扩散方程(1)的相应差分格式:

$$
C_{i} \phi_{i}=C_{i-1} \phi_{i-1}+C_{i+1} \phi_{i+1}+C_{i}^{0} \phi_{i}^{0}+C_{i-1}^{0} \phi_{i-1}^{0}+C_{i+1}^{0} \phi_{i+1}^{0}+C_{s} C_{i}+\delta S_{i},
$$

其中

$$
\begin{aligned}
& C_{i}=2(6 r+5), \\
& C_{i-1}=(6 r-1) \phi(h), \\
& C_{i+1}=(6 r-1) \psi(-h), \\
& C_{i}^{0}=2(5-6 r) \varphi(\tau), \\
& C_{i-1}^{u}=(6 r+1) \phi(h) \varphi(\tau), \\
& C_{i+1}^{0}=(6 r+1) \phi(-h) \varphi(\tau), \\
& C_{t}=12 r h^{2}, \\
& \delta S_{i}=\frac{r}{D} h^{4}\left\{(1-2 r)\left[\left(\frac{\partial^{2} S}{\partial x^{2}}\right)_{i}-\frac{U_{i}}{D}\left(\frac{\partial S}{\partial x}\right)_{i}\right]-\frac{6 r}{D}\left(\frac{\partial S}{\partial t}\right)_{i}\right. \\
& \left.\quad \quad+(1-6 r)\left[\left(\frac{U_{i}}{2 D}\right)^{2}-\frac{1}{2 D}\left(\frac{\partial U}{\partial x}\right)_{i}\right] S_{i}\right\} .
\end{aligned}
$$

显然,差分格式充分地体现了对流的“迎风”效应和“历经”效应：上游的贡献恒大于下游贡献, 随之 $|U|$ 的增加, 上游贡献 $\left(C_{i-1} 、 C_{i-1}^{0}, U>0 ; C_{i+1} 、 C_{i+1}^{0}, U<0\right)$ 加大, 下游的减小且 渐趋于零,此乃对流“迎风”效应实质; 同时, 时间步长愈大, 上时段贡献 $\left(C_{i}^{0} 、 C_{i-1}^{0}\right.$ 和 $\left.C_{i+1}^{0}\right)$ 亦 愈小, 此所谓“历经”效应使然. 故而, 差分格式确保了差分解与微分解的定性相符. 此外, $S=0$ 时, $\phi$ 与 $\phi+$ const. 均为方程(1)的解, 差分方程(15)亦反应这一特性, 就要求差分系 数满足关系式:

$$
C_{i}-C_{i-1}+C_{i+1}+C_{i}^{0}+C_{i-1}^{0}+C_{i+1}^{0}
$$

为对离散所带来偏差有所纠正 ${ }^{\mathrm{t} 33}$, 我们以式(17)取代式 (16.1), 即系数 $C_{i}$ 确定为

$$
\begin{aligned}
& C_{i}=(6 r-1)[\psi(h)+\psi(-h)]+2(5-6 r) \varphi(\tau) \\
&+(6 r+1) \varphi(\tau)[\psi(h)+\psi(-h)] .
\end{aligned}
$$

差分方程 (15) 的等价微分方程为

$$
\frac{\partial \phi}{\partial z}+U \frac{\partial \phi}{\partial x}-D \frac{\partial^{2} \phi}{\partial x^{2}}+S+O\left(\sum_{i=0}^{3} h^{2 i} \tau^{2-i}\right) .
$$

可见差分格式具有 $O\left(\sum_{i=0}^{3} h^{2 i} \tau^{2-i}\right)$ 阶精度, $\tau-O\left(h^{2}\right)$ 时, 精度四阶 $O\left(h^{4}\right)$.

\section{3 格式的稳定性}

采用 Fourier 分析方法, 对任一 $k$ 波型 Fourier 分量, 格式(15)的就动误差放大系数

$$
G-\frac{C_{i}^{0}+C_{i-1}^{0} \mathrm{e}^{-i k h}+C_{i+1}^{0} \mathrm{e}^{i k h}}{C_{i}-C_{i-1} \mathrm{e}^{-i k h}-C_{i+1} \mathrm{e}^{i k h}} .
$$

考虑到式 (17) 或 (18), $G$ 之模 $|G| \leqslant 1$ 恒成立, 故格式具有无条件稳定性. 


\section{4 算例}

构造 $\frac{\partial \phi}{\partial t}+\frac{\partial \phi}{\partial x}=\frac{\partial^{2} \phi}{\partial x^{2}}-\mathrm{e}^{x-t}(0<x<1, \imath>0)$ 其解析值 $\phi=\mathrm{e}^{x-t}$ 以求对格式进 行基本数值验证; 为比较有据, 同时选用现流行一阶迎风格式为参考. 表 1 给出了不同网格最

表 1 最大计算误差变化

\begin{tabular}{|c|c|c|c|c|c|c|}
\hline \multirow{2}{*}{ 时间 } & 本文格式 & 参考格式 & 本文格式 & 参考格式 & 本文格式 & 参考格式 \\
\hline & \multicolumn{2}{|c|}{$h=0.2, \tau=0.04$} & \multicolumn{2}{|c|}{$h=0.1, \tau=0.01$} & \multicolumn{2}{|c|}{ 误差缩小倍数 } \\
\hline 0.04 & $0.116825 \times 10^{-4}$ & $0.628221 \times 10^{-2}$ & $0.550313 \times 10^{-6}$ & $0.320045 \times 10^{2}$ & 21.2 & 1.96 \\
\hline 0.20 & $0.281333 \times 10^{-4}$ & $0.159469 \times 10^{-1}$ & $0.131130 \times 10^{-3}$ & $0.810062 \times 10^{-2}$ & 21.4 & 1.97 \\
\hline 0.40 & $0.267028 \times 10^{-4}$ & $0.157628 \times 10^{-1}$ & $0.143051 \times 10^{-3}$ & $0.773775 \times 10^{-2}$ & 18.7 & 2.03 \\
\hline 0.60 & $0.222921 \times 10^{-4}$ & $0.133615 \times 10^{-1}$ & $0.953674 \times 10^{-6}$ & $0.648498 \times 10^{-2}$ & 23.3 & 2.06 \\
\hline 0.80 & $0.184774 \times 10^{-4}$ & $0.110164 \times 10^{-1}$ & $0.894069 \times 10^{-6}$ & $0.532972 \times 10^{-2}$ & 20.6 & 2.06 \\
\hline 1.00 & $0.150799 \times 10^{-4}$ & $0.903242 \times 10^{-2}$ & $0.715255 \times 10^{-6}$ & $0.436633 \times 10^{-2}$ & 21.0 & 2.06 \\
\hline 1.20 & $0.122785 \times 10^{-4}$ & $0.739729 \times 10^{-2}$ & $0.596046 \times 10^{-6}$ & $0.357532 \times 10^{-2}$ & 20.6 & 2.06 \\
\hline 1.40 & $0.100433 \times 10^{-4}$ & $0.605675 \times 10^{-2}$ & $0.506639 \times 10^{-6}$ & $0.292721 \times 10^{-2}$ & 19.8 & 2.07 \\
\hline 1.60 & $0.819563 \times 10^{-3}$ & $0.495889 \times 10^{-2}$ & $0.447034 \times 10^{-6}$ & $0.239664 \times 10^{-2}$ & 18.3 & 2.07 \\
\hline 1.80 & $0.673532 \times 10^{-5}$ & $0.406006 \times 10^{-2}$ & $0.327825 \times 10^{-6}$ & $0.196215 \times 10^{-2}$ & 20.5 & 2.07 \\
\hline 2.00 & $0.551343 \times 10^{-5}$ & $0.332406 \times 10^{-2}$ & $0.268220 \times 10^{-6}$ & $0.160649 \times 10^{-2}$ & 20.5 & 2.07 \\
\hline
\end{tabular}

大计算误差 Max ( $\phi_{t+*}-\phi_{\text {哳 }}$ ) 随时间的变化, 显然本文格式绝对优越, 相同条件下两种格 式误差相差 600 或 6000 倍左右. 表 1 亦列出了对格式精度数值检验的结果; 据差分理论, 对 空间四阶(时间二阶)格式,空间网格缩小一倍(时间网格四倍), 其误差应减小 $2^{4}\left(4^{2}\right)=16$ 倍; 而本文格式的减小了 20 倍左右(见表 1), 充分反映了四阶格式精度特性; 参考格式仅一阶精 度,故相同条件下其误差仅缩小 2 倍. 另从表 1 中亦可看出, 本文格式粗网格的结果亦明显优 于参考格式细网格下的值, 故而本文格式实现了较粗网格下获得较藏精度数值解.

致谢 承蒙北京大学吴江航教授支持和指导,对此不胜感激。

\section{参考文献}

[1] Rai, M. M., AIAA Paper 87-0543, 1987.

[2] Roger, S. E., Kwak, D., AlAA J., 1990, 28(2): 253-262.

[3] Yang, G. L. ez al., Int. J. Num. Meth. in Fluids, 1991, 12(1): 43-58.

[ 4] Hirsh, R. S., J. Comp. Phys., 1975, 19(1): 90-109.

[5] Dennis, S. C. R., Hundson, J. D., J. Comp. Phys., 1989, 85(2): 390-416.

[6] 陈国谦、杨志峰, 中国博士后论文集, 北京大学出版社, 北京, 1991, (4): 132-141.

[7] 陈国谦、杨志峰、高智,计算物理, 1991, 8(4): 132-141.

[8] 杨志峰、周雪渵、许协庆,水动力学研究与进展, 1991，6(1): 113-119.

[9] 杨志峰、陈圂垪、罗朝俊,中国博士后论文集, 北京大学出版社, 北京, 1991, (4): 99-104。

[10] 杨志盽、周同明,北京师范大学学报(自然科学版), 1992, 28(1): 124-128.

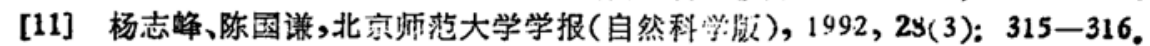

[12] 杨志峰、许协庆,水动力学䂨究与进展, 1992, 7(3): 262-268.

[13] 陈匡渞、高智,力学学报, 1991, 23(4): 418-425. 